\section{BMJ Open Sport \& Exercise Medicine}

\title{
Trends in player body mass at men's and women's Rugby World Cups: a plateau in body mass and differences in emerging rugby nations
}

\author{
Ross Tucker (D , , ${ }^{1}$ Stuart Lancaster, ${ }^{2}$ Phil Davies, ${ }^{3}$ Gary Street, ${ }^{4}$ Lindsay Starling, ${ }^{5}$ \\ Cian de Coning, ${ }^{1}$ James Brown ${ }^{6}$
}

\begin{abstract}
To cite: Tucker R, Lancaster $\mathrm{S}$, Davies $\mathrm{P}$, et al. Trends in player body mass at men's and women's Rugby World Cups: a plateau in body mass and differences in emerging rugby nations. BMJ Open Sport \& Exercise Medicine 2021;7:e000885. doi:10.1136/ bmjsem-2020-000885
\end{abstract}

Accepted 8 November 2020

\section{ABSTRACT}

Objectives This study assessed the mass of international rugby players in the men's and women's Rugby World Cups between 1991 and 2019. The objective was to quantify changes in mass of players by position, and to compare changes between men and women, and between established (Tier 1 (T1)) and emerging (Tier 2 (T2)) rugby nations.

Setting Rugby World Cups from 1991 to 2019 for men's players and 2010 to 2017 for women's players.

Participants 4447 elite male and 958 elite female players.

Primary and secondary outcome measures Player body mass, grouped as men and women, $\mathrm{T} 1$ and $\mathrm{T} 2$ nations, and by playing position, assessed over time. Results Men's player mass increased significantly between 1991 and 2019 (T1 overall 9.7\% increase), but this increase occurred almost entirely up to 2011. Women's forwards mass increased by $4.8 \%$ in $\mathrm{T} 1$, with no changes in T2 or backs from either tier. Significant differences in mass were found between $\mathrm{T} 1$ and $\mathrm{T} 2$ forwards and backs for both men and women.

Conclusions The body mass of men's players has stabilised after initial increases following professionalisation. Player body mass may be approaching a plateau, beyond which no further performance advantages occur. Changes to laws and tactical approaches by coaches may have contributed to this, by changing match demands on players, necessitating endurance, agility and speed. Trends in the evolution of T2 players suggest a barrier to identifying and developing heavy athletic players, and may require intervention to ensure competitive parity.

Ireland

${ }^{2}$ Leinster Rugby, Dublin, Ireland ${ }^{3}$ University of South Wales, Pontypridd, UK

${ }^{4}$ Harlequins Women's Rugby, London, UK

${ }^{5}$ Department for Health, University of Bath, Bath, UK ${ }^{6}$ Department of Orthopaedics, Stellenbosch University, Stellenbosch, Western Cape, South Africa

Correspondence to Dr Ross Tucker; ross.tucker@mweb.co.za

\section{INTRODUCTION}

Rugby Union is a sport involving frequent contact events, consisting of tackles, rucks, grappling situations such as mauls, scrums and collisions. ${ }^{12}$ In the modern game, approximately 200 tackles occur per match. ${ }^{3}$ Given the physicality of the sport, it is unsurprising that the strength, power and size of players are prioritised as important contributors to success by coaches.

\section{Strengths and limitations of this study}

- This is a large-scale, real-life study that evaluates every player to compete at the men's and women's Rugby World Cups since 1991 and 2010 respectively.

- Body mass was by position and within tiers to compare evolution of player mass with a view to understanding how the game may have changed over a period that spans professionalism and numerous law changes.

- The study provides novel data that inform comparative reference ranges for elite rugby players, and identifies trends in Tier 2 nations that may drive investment and interventions to ensure parity in future.

- Elite-level coaches offer novel insights and hypotheses for why observed changes have occurred.

- The data also inform potential future research and law change, since player size is a frequently discussed factor for player welfare and these data provide context for those discussions.

- Limitations include the inability to assess body composition, over and above the simple metric of body mass, which may further elucidate how professional player development has changed the physical demands of the sport. Data are provided by teams and not collected directly by the researchers.

It has previously been shown that average men's player mass has increased significantly over the last two decades, most notably in backline players. ${ }^{45}$ Hill et al documented international Northern Hemisphere players in the Five and Six Nations competition every decade from 1955 to 2015, and found that player mass remained relatively constant from 1955 to 1985, and then increased substantially beginning in 1995, coincident with the advent of professionalism in the sport. ${ }^{4}$ The result was an overall increase in average player mass of $24.3 \%$ (84.8 kg in 1955 to $105.4 \mathrm{~kg}$ in 2015), comprised of a relatively small increase between 1955 and 1995 (approximately 5\%), 
and a much larger increase of almost 20\% from 1995 to $2015 .^{4}$

No similar longitudinal tracking exists for elite women's rugby. The women's game has grown significantly in the last decade, with the first movements towards professionalism as recently as 2016. The sport remains semiprofessional and entirely amateur in many countries, and may be considered to be at a similar stage of its life cycle as men's elite rugby was in the early 1990s, though with large disparities between countries in terms of the development of professionalisation, the number of players, financial support and competition structures. Whether differences in player size between more advanced successful nations and developing nations exist is of interest for projecting the development of the women's game.

Increases in body mass, particularly recently, are of interest for both player welfare and performance reasons. Given the frequency of contact events in rugby, the contribution made by body mass to inertia, momentum and kinetic energy to injury risk ${ }^{67}$ means that collisions involving larger players, or where mismatches occur, may increase injury risk. ${ }^{8}$ This, in turn, has been suggested to threaten participation in the sport, with calls to reduce player size for safety reasons. ${ }^{910}$

Size confers advantages on larger players since it enables greater absolute force production and may improve players' ability to 'win' collisions. Advantages gained in tackles and rucks may improve ball retention ability, while forwards who are involved in so-called 'tight' or static situations such as scrums, mauls and rucks, can produce greater forces, and are less likely to be moved or pushed off the ball. It has been found that more successful teams at Rugby World Cups (RWCs) have significantly heavier forwards than less successful teams. ${ }^{11}$

At some point, however, increasing body mass may compromise acceleration, speed, agility and endurance, becoming detrimental to performance. It may be expected that an upper limit for body mass exists, beyond which other elements of performance deteriorate. This notion may be supported by the observation that backs and forwards were not significantly heavier at the 2015 RWC than the 2007 or 2011 events, ${ }^{12}$ and that professional players in England's premier competition largely stayed at the same body mass from 2002 to $2011 .^{5}$ Whether this trend has continued since 2015 is unknown.

A further consideration for the sport is that Rugby Union has been dominated by the so-called Tier 1 (T1) countries. These are nations with a significant history in the sport, professional club competitions, greater financial resources and who participate in the two premier annual international competitions, the Six Nations and the Rugby Championship. World Rugby has invested significantly in the Tier 2 (T2) nations in order to create greater competitive parity, with a significant focus on identifying and supporting the development of players from these developing rugby countries. Size disparities between $\mathrm{T} 1$ and $\mathrm{T} 2$ players have often been anecdotally offered as an explanation for the performance gap, but this has not been explored to date.

Accordingly, the aim of this study was to document the body mass of elite international men's and women's players at RWCs from the emergence of professionalism (early 1990s for men, 2010 for women) to the present day. We wished to document whether body mass has changed differently by playing position group and between rugbyplaying levels (T1 and T2). A secondary objective was to produce comparative or reference data for current generations of players in each position in men's and women's elite rugby.

\section{METHODS}

A cross-sectional study was performed using data from the World Rugby's Rugby Information Management System (RIMS). RIMS aggregates team information including the age, playing position, body mass and height of each player as squads are announced prior to each World Cup. These data are thus provided to World Rugby by teams when registering players for the tournament, and stored in the database as well as communicated to media outlets for use during tournaments.

Mass and playing position data were obtained for the Men's RWC from 1991 to 2019 (every 4years, eight tournaments) and Women's RWC in 2010, 2014 and 2017 (three tournaments). For this analysis, players were classified broadly into backs or forwards, rather than specific positions, for which data were available from 2003 onwards.

Players were grouped by tier, with countries identified as being T1 or T2. For men, T1 nations were the Six Nations teams (England, Wales, Ireland, Scotland, France and Italy) and the four nations that participate in the annual Rugby Championship event (New Zealand, Australia, South Africa and Argentina), and which have participated in every World Cup for which they were eligible $(n=10)$. T2 Nations were all other participating nations, though we chose to evaluate the Pacific Island (PI) nations (Fiji, Samoa and Tonga) as a subset of T2 nations since there is a general though unconfirmed perception that these players are larger in size than other T2 players, resulting in 12T2 nations and 3 PI teams.

For women, T1 nations were countries that have reached at least one semifinal in the 2010, 2014 and 2017 Women's RWCs ( $=7$, Australia, Canada, England, France, New Zealand, USA and Ireland) and that participated in all three events, while T2 nations were those that had never reached the semifinal stage $(n=10$, Kazakhstan, Scotland, South Africa, Wales, Samoa, Spain, Hong Kong, Italy and Japan)

Data were checked for normal distribution using a Shapiro-Wilk's test, with a $p$ value $<0.05$ rejecting the null hypothesis of a normal distribution. Non-normal data are presented as medians with IQR, and selected percentiles we evaluated to explore the distribution of player mass over time. 
The normal distribution assumption for body mass was rejected, with a Shapiro-Wilk p value $<0.05$, for many groups (table 1). Thus, changes over time within tier (T1, T2, PI) and positional (forwards, backs) groups were assessed using a Kruskal-Wallis test to compare medians between the eight men's and three women's tournaments. If the overall Kruskal-Wallis was significantly different $(\mathrm{p}<0.05)$, a Wilcoxon rank-sum test was performed in a pairwise fashion, with pairwise significance accepted at a Bonferroni-corrected $\mathrm{p}$ value $(\alpha / \mathrm{n})$, with ' $n$ ' being the number of outcomes, depending on the question. Median mass was compared within RWC tournaments between tiers groups (T1, T2, PI) using the same methods.

Reference ranges for body mass in each playing position were evaluated using percentiles to identify ranges. Body mass was categorised as broadly normal (between 25th and 75th percentile), below/above average (10th to 25th and 75th to 90th percentiles), unusually low/high (2nd to 10th and 90th to 98th percentiles) and extremely low/high (below 2nd and above 98th percentile).

The research plan for this study was approved by the World Rugby Institutional Ethics committee (REF 19007). Since no player's medical information was used, and personal data were used only in a deanonymised form, and was available publicly through online sources, informed consent was not required from players in the relevant tournaments.

\section{Patient and public involvement}

Patients or the public were not involved in the design, or conduct, or reporting or dissemination plans of the research.

\section{RESULTS}

Eight men's tournaments featuring 25 unique teams, 151 total teams (data were missing for Tonga in 1995, and for 173 individual players in the tournaments), and 4447 total players were included in the final analysis. Three women's tournaments with 12 teams each and 958 total players made up the women's cohort. We chose to include each appearance of players who appeared in multiple World Cups, since their body mass may have changed from one tournament to the next, contributing to the global or collective trend over time that we wished to explore.

Table 1 summarises the body mass of players by position group in the eight men's and three women's tournaments. Data were non-normally distributed, and all subsequent analysis evaluates medians and IQR for comparisons over time and between tier groups.

\section{Trends over time}

Figure 1 depicts the median mass of forwards and backs, grouped into tiers, in men's and women's players. Among forwards in men's World Cups (figure 1A), median mass in T1 players increased significantly from 1991 to 1995 $(p<0.0005)$. No other single tournament increases in body mass in any tier group were significant, though the cumulative effect of small increases over time was a significant increase in the median mass of forwards in all tiers between 1991 and 2019. T1 forwards' median mass increased by $9.7 \%$ (103 kg to $113 \mathrm{~kg}, \mathrm{p}<0.0005)$, T2 nations by $8.0 \%$ (100 kg to $108 \mathrm{~kg}, \mathrm{p}<0.0005)$, and PI teams by $9.5 \%$ (105 kg to $115 \mathrm{~kg}, \mathrm{p}<0.0005$, figure $1 \mathrm{~A}$, table 1).

Assessing the most recent men's RWC (2019) relative to preceding tournaments, T1 forwards had a similar median mass in 2019 compared with 2007, 2011 and 2015. T1 forwards in 2019 were however significantly heavier than T1 forwards in all RWCs up to and including 2003. In contrast, T2 teams' median forward mass in 2019 was similar to all RWCs since 2003, with the most recent significant difference observed when comparing 2019 to 1999 (108 $\mathrm{kg}$ vs $105 \mathrm{~kg}$, figure 1A). PI forwards were significantly heavier in 2019 than in the 1991 and 1995 RWCs, with no other differences detected between 2019 and any RWC since 1995.

In men's T1 backs, median mass increased at every RWC from 1991 to 2011, with the only significant single tournament increase between $1995(85 \mathrm{~kg})$ and 1999 ( $89 \mathrm{~kg}$, figure $1 \mathrm{~B}, \mathrm{p}<0.0005)$. The effect of the cumulative increases in median mass was an overall increase of $10.8 \%$ between 1991 and 2019 (83 kg to $92 \mathrm{~kg}, \mathrm{p}<0.0005$ ), achieved entirely between 1991 and 2011. Comparing 2019 to previous RWCs, T1 backs were significantly heavier than in 1991 and 1995, with no significant differences detected from 1999 onwards.

In $\mathrm{T} 2$ teams, the median mass of backs increased at every RWC from 1995 onwards, with a significant single tournament increase between 1995 (median $80 \mathrm{~kg}$ ) and 1999 (median $85 \mathrm{~kg}$ ). The overall change between 1991 and 2019 was $12.2 \%$. T2 backs in the 2019 RWC were significantly heavier than in 1999 and earlier, with no significant differences between the 2019 median and any RWC from 2003 onwards (figure 1B).

PI backs' median mass in 2019 was significantly greater than in 1991 (94 kg vs $85 \mathrm{~kg}, 11.2 \%$ increase, $\mathrm{p}<0.0005$ ), though no significant differences were found for single tournament increases, or between 2019 and any previous RWCs since 1991.

In women, the median mass of $\mathrm{T} 1$ forwards increased by $4.8 \%$ from 2010 to 2017 ( $79 \mathrm{~kg}$ in 2010 to $83 \mathrm{~kg}$ in 2017, $\mathrm{p}<0.001$ ), though no single tournament changes were significant. In contrast, the median mass of T2 forwards was unchanged between 2010 and 2017 (75 kg to 73 $\mathrm{kg})$, with a tendency for a significant decrease between 2014 and 2017 ( $76 \mathrm{~kg}$ to $73 \mathrm{~kg}, \mathrm{p}=0.0341$, figure 1C). No changes were observed in the median mass of women's backs for either T1 or T2 nations (figure 1D).

\section{Comparison between tiers}

Comparing forwards' mass between tier groups at the same RWC, T1 nations had heavier forwards than T2 nations in 2019 (113 kg vs $108 \mathrm{~kg}, \mathrm{p}<0.0005)$, while PI teams forwards were significantly heavier than T2 


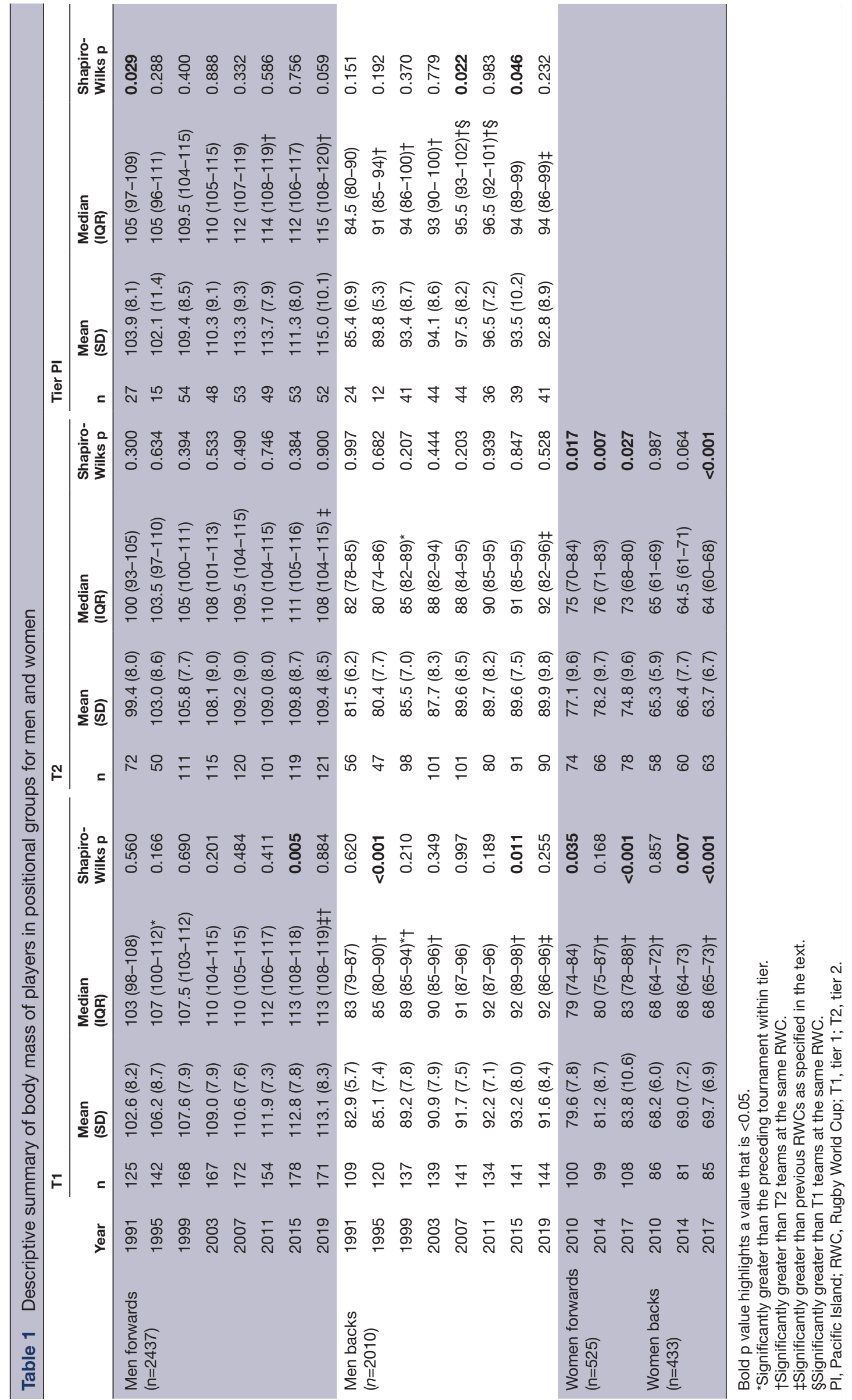


A) Men's forwards
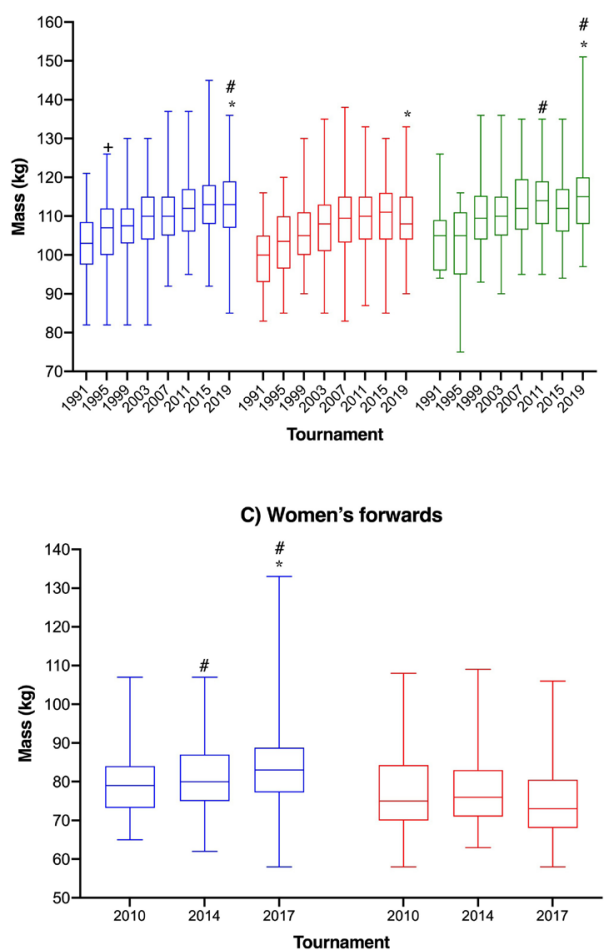

B) Men's backs

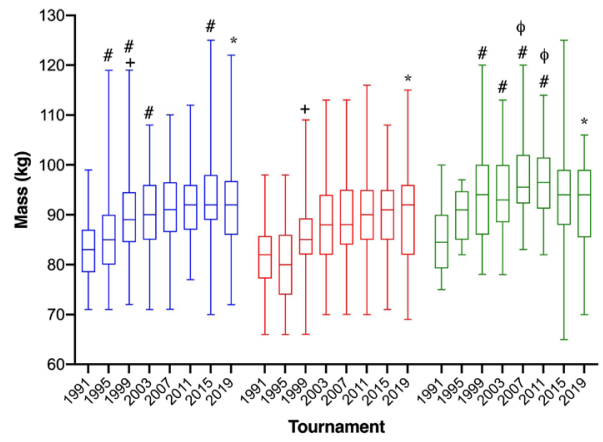

D) Women's backs

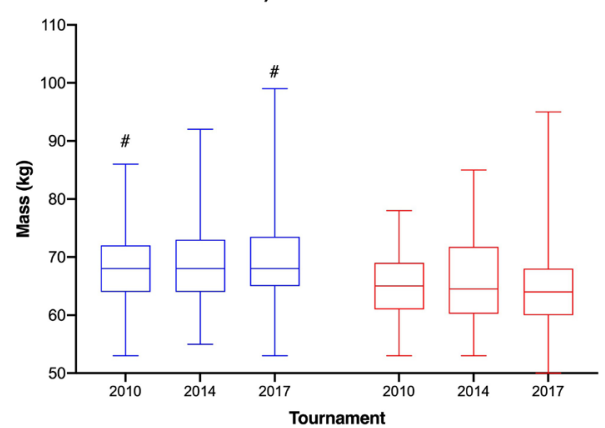

Figure 1 Evolution of the mass of forwards (left column) and backs (right column) of players in men's (top row) and women's (bottom row) RWCs. Data are shown from 1991 to 2019 for men, with nations grouped into T1, T2 and PI nations, while women's data are for 2010 to 2017, grouped into T1 and T2 nations. Data presented as medians (IQR and range). ${ }^{+}$Significantly greater than the preceding tournament within tier. "Significantly greater in 2019 compared with previous tournaments specified in text. "Significantly greater than in T2 teams at the same RWC. ${ }^{\$}$ Significantly greater than T1 teams at the same RWC. PI, Pacific Island; RWC, Rugby World Cup; T1, Tier 1; T2, Tier 2.

forwards in 2011 and 2019 ( $\mathrm{p}<0.0005$, table 1). In backs, $\mathrm{T} 1$ players were significantly heavier than $\mathrm{T} 2$ players in the tournaments between 1995 and 2003, and in 2015, while PI backs were significantly heavier than T2 backs from 1995 to 2011 (table 1). PI backs were also heavier than T1 backs in 2007 and 2011 (figure 1B).

As a result of the increase in body mass of $\mathrm{T} 1$ forwards over time, they were significantly heavier than T2 forwards in 2014 and 2017 (figure 1C, $\mathrm{p}<0.0005$ ), and T1 backs were significantly heavier than T2 backs in 2010 and $2017(\mathrm{p}<0.0005)$.

Figure 2 summarises the relative changes in median mass in consecutive tournaments in forwards and backs within each tier group. The ratio is calculated as median mass of $T_{n}$ divided by median mass of $T_{n-1}$ where $n$ is a given RWC number within the cohort. The largest increases (change 1.03-fold or higher) occurred in the first three tournaments for men (1991 to 2003), with
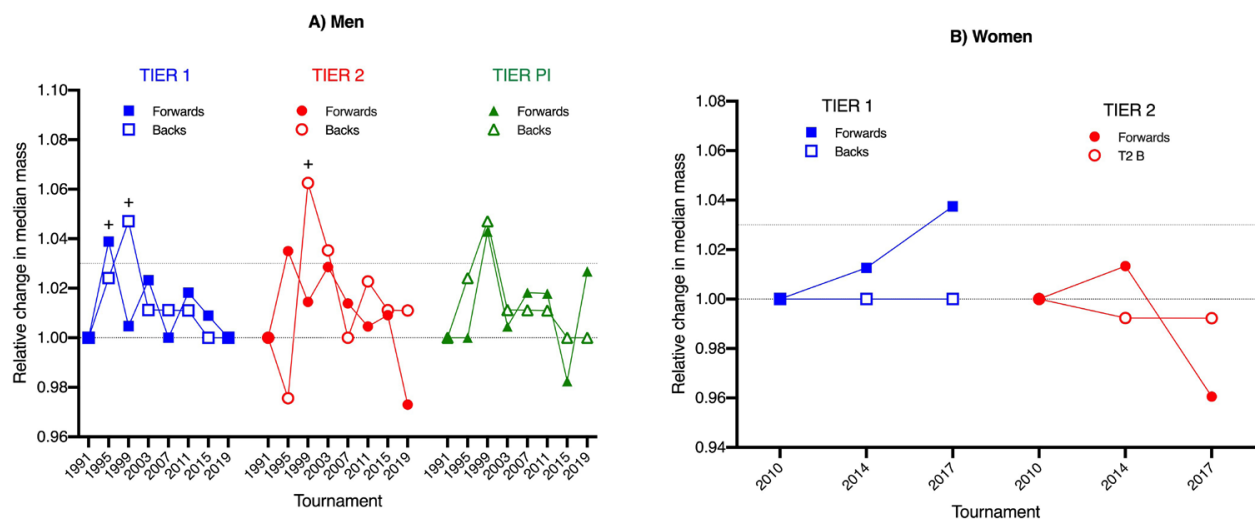

Figure 2 Relative change in median mass in consecutive tournaments. Change is calculated as median mass of T/median mass $T_{n-1}$. Dashed lines indicate no change (1.0) and a 1.03-fold increase compared with the previous tournament. Forwards are indicated by solid symbols, while backs are shown as open symbols for the tier groups. ${ }^{+}$Significant increase in median mass compared to the preceding tournament within tier group. 
A) Men's Forwards

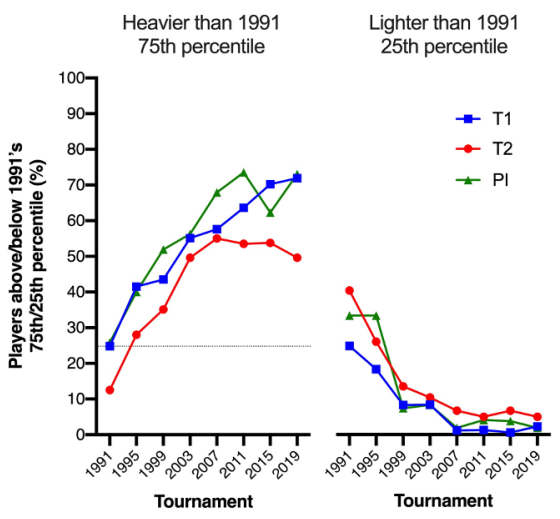

C) Women's Forwards

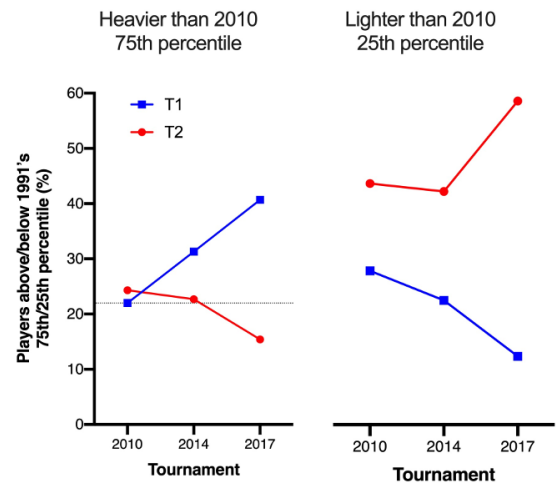

B) Men's Backs

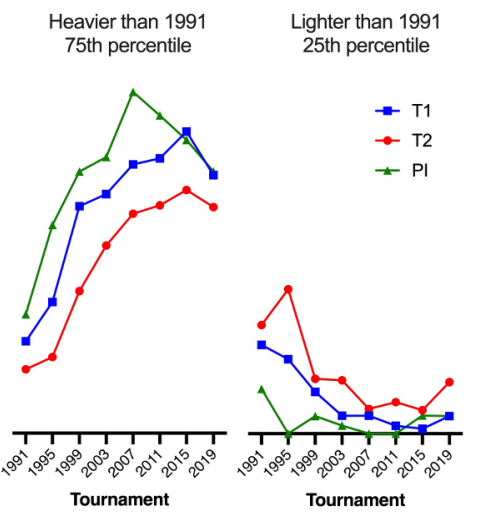

D) Women's Backs

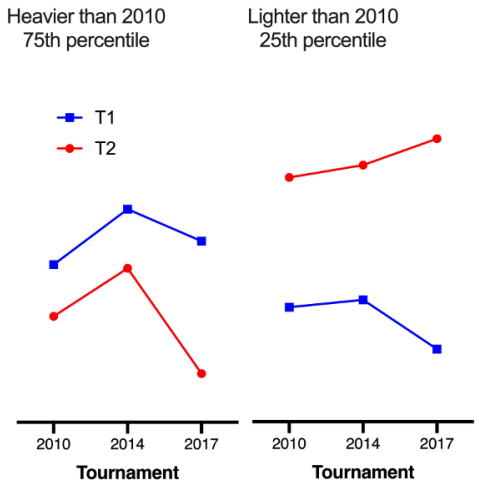

Figure 3 Historical evolution of the proportion of players whose mass is greater than the 75th percentile and less than the 25th percentile of T1 players at the first tournament in the cohort. The dashed line indicates the proportion of T1 players whose mass is greater than the 75th percentile the 1991 men's RWC (A and B) and the 2010 women's RWC (C and D). PI, Pacific Island; RWC, Rugby World Cup; T1, Tier 1; T2, Tier 2.

no increases above 3\% since 2003. For women, 2017 increases in forward mass of $\mathrm{T} 1$ players reached $3 \%$, whereas $\mathrm{T} 2$ forwards and backs decreased in body mass.

To further examine differences in the body mass of players over time, we explored how player mass evolved with reference to a baseline value corresponding to the heaviest and lightest quartile of T1 players from the 1991 tournament (men) and 2010 tournament (women). The 25th and 75th percentiles for $\mathrm{T} 1$ forwards and backs were first determined and then used as reference values to calculate the proportion of players heavier than that 75 th percentile and lighter than that 25th percentile at each subsequent tournament to be calculated. These proportions are depicted in figure 3.

\section{Evolution of heaviest quartile of forwards}

For men's forwards, the 75th percentile in 1991 was $108 \mathrm{~kg}$, with $24.8 \%$ of $\mathrm{T} 1$ players heavier than this body mass. In $\mathrm{T} 1$ teams, the proportion of forwards heavier than $108 \mathrm{~kg}$ increased at every subsequent tournament, peaking in 2019, where $71.9 \%$ of players were heavier than the reference value (figure 3A). A similar increase was observed for PI forwards, of whom $73.1 \%$ were heavier than $108 \mathrm{~kg}$ in 2019. The proportion of T2 forwards heavier than $108 \mathrm{~kg}$ increased up to $2007(55.0 \%)$, but has since stabilised and declined, with only $49.6 \%$ of T2 forwards heavier than $108 \mathrm{~kg}$ in the 2019 RWC. At every RWC, the proportion of $\mathrm{T} 2$ forwards heavier than the reference body mass of $108 \mathrm{~kg}$ was lower than in $\mathrm{T} 1$ forwards, though the gap narrowed between 1995 (41.5\% of T1 vs $28.0 \%$ of T2 above $108 \mathrm{~kg}$ ) and $2007(57.6 \%$ of T1 vs $55.0 \%$ of T2 above $108 \mathrm{~kg}$ ), before increasing after 2007, with the largest gap between $\mathrm{T} 1$ and $\mathrm{T} 2$ players recorded in 2019 (71.9\% of T1 forwards vs $49.6 \%$ of $\mathrm{T} 2$ forwards heavier than $108 \mathrm{~kg}$, figure $3 \mathrm{~A}$ ).

\section{Evolution of lightest quartile of forwards}

The opposite trends occurred with reference to the lightest quartile of T1 forwards in 1991 (98 kg, with 24.8\% of $\mathrm{T} 1$ forwards lighter than this body mass, figure $3 \mathrm{~A}$ ), with a trend for this proportion to decrease over time, indicative of fewer lighter players over time. The proportion of T1 players lighter than $91 \mathrm{~kg}$ decreased at each subsequent tournament, reaching $8.3 \%$ by $1999,1.2 \%$ by 2007 and a lowest value of $0.6 \%$ in 2015 , before increasing to $2.3 \%$ in 2019. A greater proportion of $\mathrm{T} 2$ than $\mathrm{T} 1$ forwards were lighter than $91 \mathrm{~kg}$ in 1991 (40.3\% in T2, figure $3 \mathrm{~A}$ ). This proportion has decreased over time, but 
remained higher than the T1 proportion at every RWC (between $5.0 \%$ and $6.7 \%$ since the 2007 RWC, compared with $0.6 \%$ to $2.3 \%$ of T1 forwards). The proportion of PI forwards lighter than $91 \mathrm{~kg}$ decreased over time and is generally similar to that of T1 teams.

\section{Evolution of heaviest quartile of backs}

Among backs, a similar increase in the proportion of players heavier than the 75th percentile from 1991 was observed for the three-tier groups. The 75th percentile reference value for T1 backs in 1991 was $87 \mathrm{~kg}$, a body mass that was exceeded by $72.2 \%$ of T1 backs in 2019 . About $73.2 \%$ of PI backs were above $87 \mathrm{~kg}$ in 2019 , while $63.3 \%$ of $\mathrm{T} 2$ backs exceeded the $87 \mathrm{~kg}$ reference value. As occurred in forwards, the proportion of $\mathrm{T} 2$ backs heavier than the reference value decreased in 2019 compared with 2015 (figure 3B). The peak of heavy backline players for $\mathrm{T} 1$ and $\mathrm{T} 2$ teams occurred in 2015 , where $84.4 \%$ and $68.1 \%$ of T1 and T2 players were heavier than $87 \mathrm{~kg}$. For PI backs, a peak occurred in 2007 , with $95.5 \%$ of players heavier than the 1991 reference value, followed by a progressive decline to 2019 (figure 3B).

\section{Evolution of lightest quartile of backs}

With reference to the lighter players, the 25th percentile in T1 backs in 1991 was $79 \mathrm{~kg}$. The proportion of T1 backs lighter than $79 \mathrm{~kg}$ decreased progressively from 1995 to 2015, reaching $1.4 \%$ (2 out of 141 players), before increasing to $4.9 \%$ (7 out of 144 backs) in 2019 . $\mathrm{T} 2$ and PI backs show similar trends for a decrease in the number of lighter players. In 2007 and 2011, no backs from PI teams were lighter than $79 \mathrm{~kg}$. The proportion of T2 players lighter than $79 \mathrm{~kg}$ decreased from $40.4 \%$ in 1995 to $6.6 \%$ in 2015 , before increasing to $14.4 \%$ in 2019 (figure 3B), and was greater than the proportion of players lighter than $79 \mathrm{~kg}$ in T1 teams at every analysed tournament.

\section{Evolution of heaviest and lightest quartile for women}

In women, the 75th percentile of T1 forwards in 2010 was $84 \mathrm{~kg}$ (22\% of $\mathrm{T} 1$ forwards). The proportion of $\mathrm{T} 1$ forwards heavier than $84 \mathrm{~kg}$ increased in 2014 (31.3\%) and $2017(40.7 \%)$. In contrast, the proportion of forwards heavier than $84 \mathrm{~kg}$ in T2 teams decreased from $24.3 \%$ in 2010 to $15.4 \%$ in 2017 (figure 3C). The reference value for the lightest quarter of players in 2010 was $74 \mathrm{~kg}$. The proportion of T1 forwards lighter than $74 \mathrm{~kg}$ decreased to $20.2 \%$ in 2014 and $11.1 \%$ in 2010 . In contrast, the proportion of T2 forwards lighter than $74 \mathrm{~kg}$ has increased between 2010 (39.2\%) and 2017 (52.6\%), and is greater than in $\mathrm{T} 1$ forwards (figure $3 \mathrm{C}$ ).

In women's backs, the proportion of $\mathrm{T} 1$ players heavier than $72 \mathrm{~kg}$ (75th percentile in 2010) increased to $34.6 \%$ in 2014 and then decreased to 29.4\% in 2017 (figure 3D). In T2 backs, the proportion of players heavier than $72 \mathrm{~kg}$ increased from $17.2 \%$ to $25.0 \%$ in 2014 , and then decreased to $7.9 \%$ in 2017. The proportion of $\mathrm{T} 1$ backs lighter than the 25th percentile in $2010(64 \mathrm{~kg})$ decreased from $18.6 \%$ in 2010 to $11.8 \%$ in 2017 , and was lower than the proportion of T2 backs lighter than $64 \mathrm{~kg}$ in all three tournaments. The proportion of T2 backs lighter than $64 \mathrm{~kg}$ increased progressively from $39.7 \%$ in 2010 to $46.0 \%$ in 2017 (figure 3D).

\section{Country comparison}

Differences between $\mathrm{T} 1$ and $\mathrm{T} 2$ nations were explored by assessing which participating nations lay above or below the median, and in the top and bottom 10th percentiles in the three most recent RWCs for men and women. The median and IQR was calculated for the whole group ( $\mathrm{n}=60$ men's teams and 36 women's teams), with countries whose players' average masses lay below or above the 50th percentile, below the 10th percentile (lightest teams) and above the 90th percentile (heaviest teams) identified. The findings are presented in figure 4 .

Twenty out of $30 \mathrm{~T} 1$ teams had an average forwards mass above the median (67\%), compared with $44 \%$ of PI teams (4 out of 9 ) and $29 \%$ of T2 teams (6 out of 21 ). When assessing T1 versus T2 differences (excluding PI teams) for men's forwards (figure 4A), T1 teams were significantly more likely to lie above the median mass than T2 teams (OR 5.0, 95\% CI 1.5 to 16.8). Of the six teams above the 90 th percentile for forwards, four were $\mathrm{T} 1$ teams, two PI teams and no T2 teams were among this heaviest group. The teams that lay below the 10th percentile were comprised entirely of $\mathrm{T} 2$ teams (figure 4A). The median T2 forward was similar in body mass to the 25th percentile of all teams' forwards $(109.7 \mathrm{~kg}$ vs $110.1 \mathrm{~kg}$ ).

In men's backs, $60 \%$ (18 out of 30 ) of T1 teams had an average mass above the overall median, compared with $89 \%$ ( 8 out of 9 ) of PI teams, and 19\% (4 out of 21) T2 nations. T1 nations were significantly more likely to be in the heavier median than T2 nations (OR 6.4, 95\% CI 1.7 to 23.7), and T2 nations contributed five of the six teams that lay below the 10th percentile for overall backs' mass (figure 4B).

In women, all four teams whose average forward mass was above the 90th percentile were $\mathrm{T} 1$ nations (figure 4C). About $67 \%$ of T1 teams had forwards with an average mass greater than the overall median, compared with $27 \%$ of T2 teams. T1 teams were thus significantly more likely to be above the median forward mass than T2 teams (OR 5.5, 95\% CI 1.3 to 23.7).

Among backs at the last three women's RWCs, a similar distribution was observed, with the lightest backs coming from the T2 group, while three of the four heaviest teams were $\mathrm{T} 1$ teams (figure 4D). The odds of a T1 nation having a body mass above the median were significantly greater than for T2 teams (OR 10.0, 95\% CI 2.1 to 48.6).

\section{Comparative reference ranges}

Comparative reference ranges were determined for the last three men's RWCs ( $\mathrm{n}=1794$ players) and two Women's RWCs ( $\mathrm{n}=640$ players), because it had been observed that the median mass of men's players had stopped increasing from 2011 onwards (figure 1). We 
A) Men's Forwards mass by nation last 3 RWCs

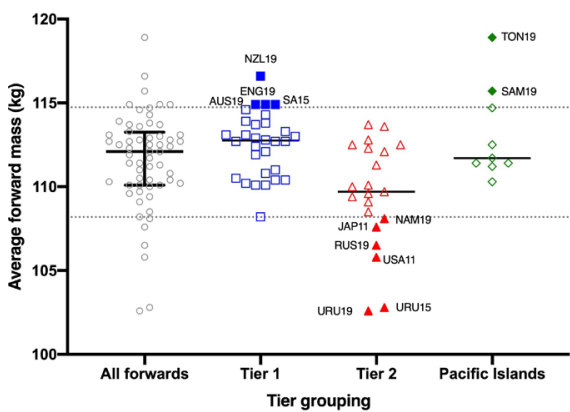

C) Women's Forwards mass by nation last 3 RWCs

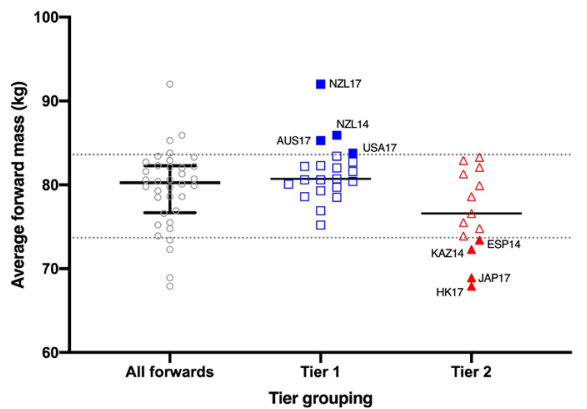

B) Men's backs mass by nation last 3 RWCs

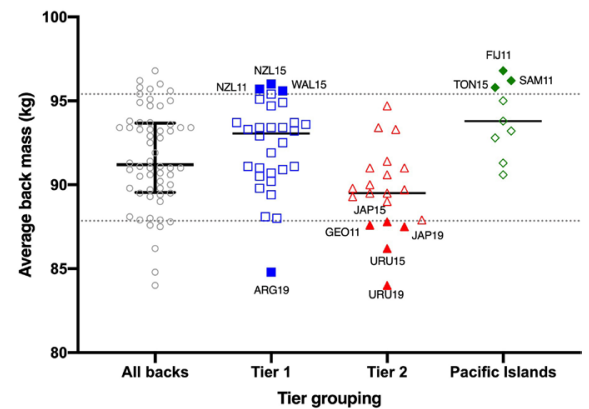

D) Women's backs mass by nation last 3 RWCs

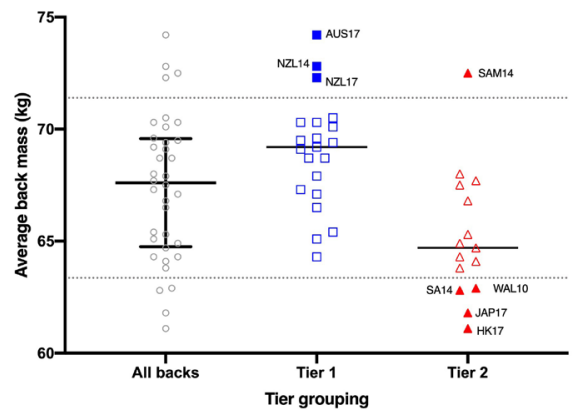

Figure 4 Average mass of forwards and backs mass for each participating team at the three most recent RWCs for men (top panels) and women (bottom panels). Teams are grouped by playing position and tier. solid lines indicate the median within each grouping, with the IQR calculated and shown for the whole group. The 10th and 90th percentiles for the whole group are shown by dashed lines. Solid symbols indicate teams below and above the 10th and 90th percentiles, respectively. RWC, Rugby World Cup.

also included all players, despite the previously described differences between T1, T2 and PI players, since this analysis is intended to provide an overview of the whole international game, inclusive of all players.
Figure 5 shows the frequency histogram for the body mass of forwards (left panel) and backs (right panel), with the dotted lines indicating the median and dashed line the 98th percentile (corresponding to extremely heavy)
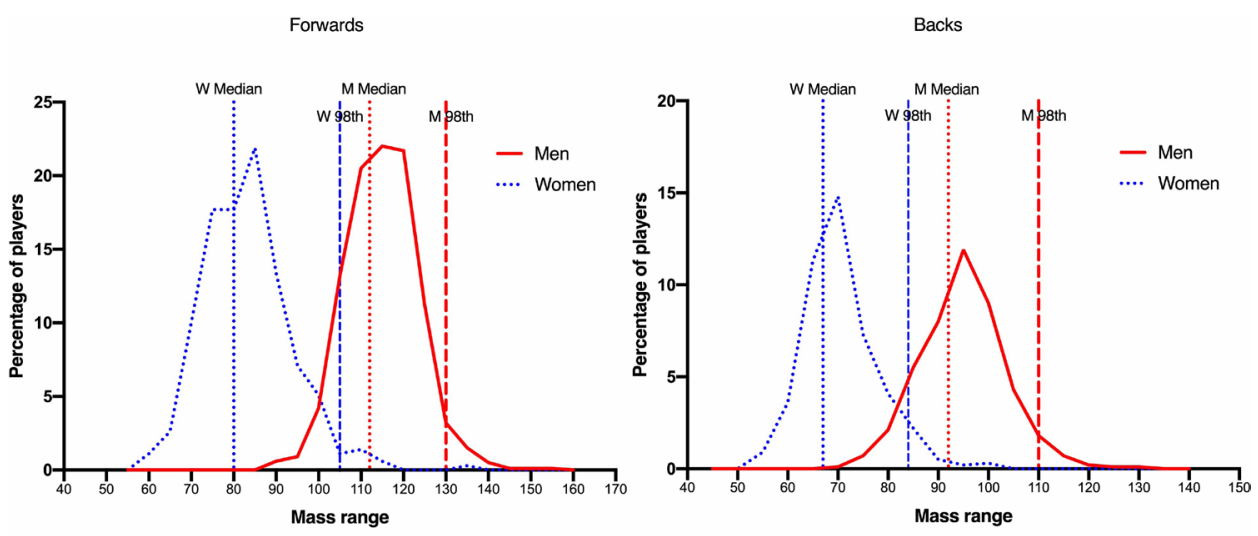

\begin{tabular}{|c|c|c|}
\hline Percentiles & Men ( $\mathbf{n = 9 9 8 )}$ & Women ( $\mathbf{n = 3 5 1 )}$ \\
\hline $\mathbf{1}$ & $\mathbf{9 2}$ & 59 \\
\hline $\mathbf{2}$ & $\mathbf{9 5}$ & 63 \\
\hline $\mathbf{5}$ & $\mathbf{9 9}$ & 66 \\
\hline $\mathbf{1 0}$ & 101 & 68 \\
\hline $\mathbf{2 5}$ & 106 & 73 \\
\hline $\mathbf{5 0}$ & 112 & 80 \\
\hline $\mathbf{7 5}$ & 118 & 85 \\
\hline $\mathbf{9 0}$ & 122 & 93 \\
\hline $\mathbf{9 5}$ & 125 & 97 \\
\hline $\mathbf{9 8}$ & 130 & 105 \\
\hline $\mathbf{9 9}$ & 132 & $\mathbf{1 0 9}$ \\
\hline
\end{tabular}

\begin{tabular}{|c|c|c|}
\hline Percentiles & Men ( $\mathbf{n = 7 9 6 )}$ & Women ( $\mathbf{n = 2 8 9 )}$ \\
\hline $\mathbf{1}$ & $\mathbf{7 2}$ & 53 \\
\hline $\mathbf{2}$ & 75 & 55 \\
\hline $\mathbf{5}$ & $\mathbf{7 8}$ & 56 \\
\hline $\mathbf{1 0}$ & $\mathbf{8 1}$ & 60 \\
\hline $\mathbf{2 5}$ & $\mathbf{8 6}$ & 63 \\
\hline $\mathbf{5 0}$ & 92 & 67 \\
\hline $\mathbf{7 5}$ & $\mathbf{9 7}$ & 72 \\
\hline $\mathbf{9 0}$ & 102 & 77 \\
\hline $\mathbf{9 5}$ & 105 & 81 \\
\hline $\mathbf{9 8}$ & 110 & 84 \\
\hline $\mathbf{9 9}$ & 112 & 89 \\
\hline
\end{tabular}

Figure 5 Frequency histograms for mass of forwards (left panel) and backs (right panel) in elite men's and women's rugby players. Dotted lines indicate the 50th percentile, while dashed lines indicated the 98th percentile for each group. 
for men and women. The median mass of forwards was $112 \mathrm{~kg}$ for men and $80 \mathrm{~kg}$ for women, while the median back player weighed $92 \mathrm{~kg}$ for men and $67 \mathrm{~kg}$ for women. The reference ranges identified in men and women for each playing position are presented in table 2 .

\section{DISCUSSION}

This study evaluated the body mass of elite international players at RWCs from 1991 to 2019 (men) and 2010 to 2017 (women). Our aims were to expand on previous findings of increased body mass after the sport became professional, with specific reference to whether developing rugby nations (T2 nations) and women have followed a similar trajectory to those observed for established T1 nations.

\section{Time trends in men}

Accordingly, our first finding is that various elements of the body mass of players in men's RWCs has stabilised in recent RWC tournaments, suggesting that an upper limit in body mass is being approached, and may even have been surpassed. This assertion is based on numerous findings, including our finding that no increases in median mass have occurred since 2011, the first documented decrease in player mass in the 2019 tournament, and our finding that the proportion of heavy players (assessed as players heavier than the top quartile from 1991) has begun to stabilise, while the proportion of lighter players (assessed as players lighter than the bottom quartile from 1991) has increased in 2019 compared with 2015.

This confirms previous research showing large increases soon after professionalism ${ }^{4}$ followed by a levelling off in body mass, ${ }^{45}$ but extends those previous findings to the most recent RWC in 2019, and explores the time-course of the increases at each RWC rather than over decades. ${ }^{4}$

We do acknowledge that single tournament increases may be small and insignificant, but their cumulative effect may be a significant increase in body mass over longer periods, as we find historically (figure 1). It cannot thus be discounted that the small increase in forwards' mass between 2011 and 2019 will continue in the future, and so it would be premature to conclude definitively that an upper limit has been reached. However, 2019 was the first RWC since 1995 where neither the forwards or backs have increased in body mass. In combination with data from the club game, we suggest that elite player mass is close to reaching levels beyond which performance advantages no longer accrue, if it has not already reached that level.

Historical comparisons between 2019 and 1991 reveal striking differences. We show, for example, that the heaviest quarter of players in 1991 would be among the lightest quarter of players in 2019 (figure 3A,B). These changes, at the heavier end of the player mass spectrum, are mirrored by similar reductions in the proportion of players lighter than a reference mass corresponding to the lightest quarter of players in 1991 (figure 3A,B), the result of which is an overall increase of $10 \mathrm{~kg}$ and $9 \mathrm{~kg}$ in the median mass of $\mathrm{T} 1$ forwards and backs, respectively.
However, these increases were largely achieved between 1991 and 2003, with much smaller cumulative and singletournament increases since.

The reasons for the body mass increases soon after professionalisation of rugby have been discussed previously. ${ }^{45}$ They include both direct and indirect consequences of professional rugby. In addition to what has been discussed previously, we offer the following coachdriven insights and hypotheses for our findings.

Directly, professional rugby has enabled players to access full-time team environments to maximise and optimise training and nutrition modalities to increase muscle mass to develop strength and power required for performance. Elements of these professional environments exist from junior levels, including talent identification systems and age-grade rugby competitions that preferentially select players with desired physical attributes. Full-time environments, including academies that were established by many professional clubs in around 2000 enable an earlier start to conditioning programmes, the effects of which have been documented as increases in body mass in 18-year-old players ${ }^{13}$ that are similar in magnitude to those described here. At some point, however, the benefits of full-time conditioning and the application of expertise will be realised, and only very small increases will be possible thereafter.

Indirectly, numerous laws have been introduced that may have facilitated the evolution of larger players. Among them was a progressive increase in the number of substitutes allowed during matches, which has allowed coaches to prepare players for shorter periods of higher intensity play. This may favour the development of power and strength, enabling greater mass to be carried without the negative performance consequences of premature fatigue. ${ }^{4}$

More recently, however, changes in the sport may have counteracted these early changes. Law changes aimed at improving aesthetics of the sport have caused ball in play time, which averaged $35 \%$ in 1995 , to increase to $44 \%$ in $2019,{ }^{2}$ and has been documented as high as $50 \%$ in recent professional club and international events. ${ }^{3}$ Combined with fewer stoppages for set pieces in the game, the result has been an increase in the length of activity cycles, particularly since $2011 .^{23}$

Coaches systematically evaluate these game demands, and then prepare players against known targets, which may have shifted conditioning paradigms towards the development of players able to withstand fatigue. A secondary consequence of this might be smaller players who carry less potentially detrimental body mass. We suggest that further increases in player size may not be possible given the game demands on players, unless future law change allows longer periods of recovery (eg, prior to set pieces, while kicks are taken, or during Television Match Official decisions) or less ball in play time. These hypotheses may be tested in future by exploring specific player involvement in various match activities, playing time by playing position and activity cycle changes over time. 


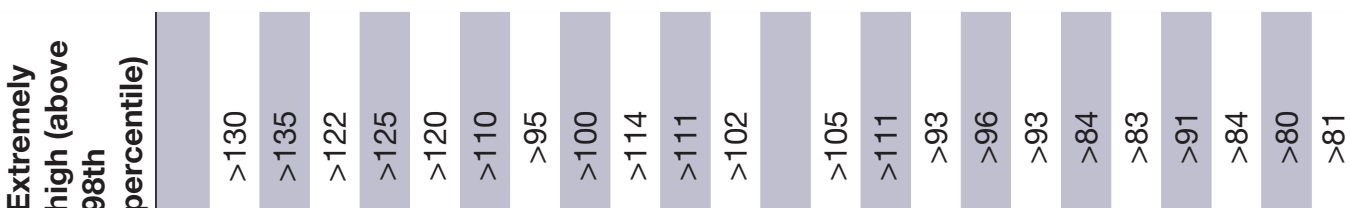
흘

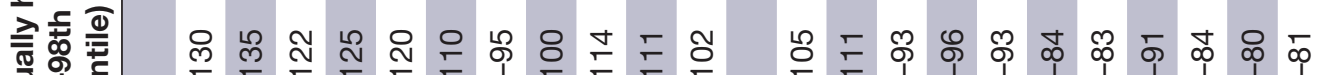

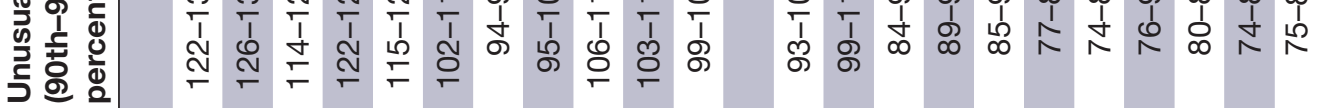
$\overline{\bar{g}}$ 을 웅

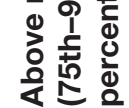

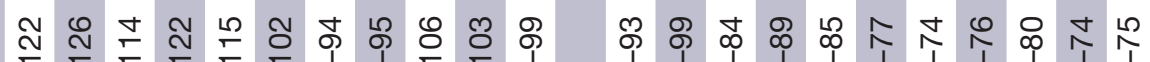
D.

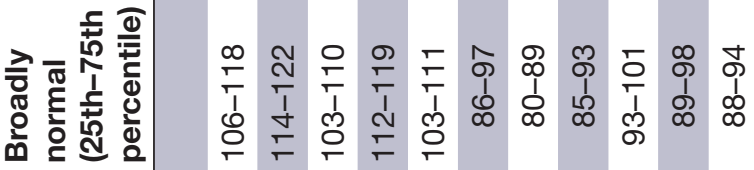

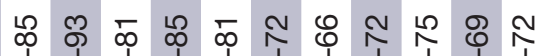

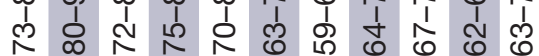

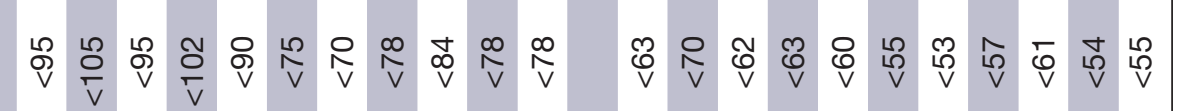


Another set of changes that may create a ceiling for player size is related to coaching intent in the sport. This encompasses both attacking and defensive strategies and tactics. It includes the desire to play continuously and at a higher pace with shorter recoveries, to seek competitive advantages through speed, fitness and playing tempo, rather than a reliance on size alone. This would necessitate a greater endurance component of conditioning, to perform constant high-tempo play. The change in tactical intent is not universal to all teams and coaches, but is generally supported by the observation that the number of passes and rucks has increased by $15 \%$ since 2007, in part due to more ball in play time, but also driven by an increase in the rate of these activities, ${ }^{2}$ as well as our hypothesis that teams may regularly retain the ball through multiple phases more frequently than in the past.

Defensively, the emergence of so-called rush-defence patterns, and teams' approaches to contesting for the ball during rucks, may also have shifted optimal size down compared with in the past. The priority of modern rugby defensive systems is to apply pressure to attacking teams by reducing space and time through aggressive, fast-rushing defensive lines. This requires that players avoid commitment to rucks, instead returning to position in the defensive line, followed by greater acceleration and speed in repeat-bouts within their defensive strategy. The ability to meet these match demands may be constrained if excessive mass is carried. Therefore, it may be that the requirement for sustained higher intensity effort has set a ceiling on size, particularly in backline players in whom mass is not required for static, high-force generating activities, and that this limit is close to being reached.

From a player welfare perspective, the increasing size of players has been named as a primary contributor to the significant injury risks documented in various injury surveillance studies in Rugby Union. ${ }^{1} 67121415$ Given that most injuries occur in the tackle, ${ }^{171617}$ with rucks second most frequently responsible for injuries, the increasing body mass of players at all levels ${ }^{411} 1318$ is of concern because contact injuries are the result of the transfer of kinetic energy and excessive inertial loading. ${ }^{7} 1920$

It would appear that such concerns had specific merit from 1995 to 2011, but that given the stabilisation of size since 2011, any impact of body mass on welfare is similar in 2019 as in 2011. Thus, while the greater size of players in 2019 is arguably a welfare consideration for comparisons between the current situation and 2003 and earlier, it is less of a factor since 2011. That said, we do recognise that body mass is a crude metric with which to understand how the physical capacity of players has changed, and that body composition, and also the ability to exert force in contact, are likely contributors to injury risk. We do not currently have measures to explore these possibilities.

\section{Time trends in women}

Among women, we have insufficient data to allow such long-term evaluation, since it was possible to evaluate body mass over only three tournaments. Within this narrow range, we find variable increases in body mass from tournament-by-tournament (figure 2). Women's rugby is still amateur and semiprofessional in most nations, with the first elements of professionalism, including full-time salaried players, access to regular high-level club competition and the application of sports science and medicine, introduced only in 2016.

In this regard, some nations in women's international rugby are at a stage of their life cycle similar to the men's game in 1995. While it would be premature to predict that the trend among women will follow what was observed in men between 1995 and 2003, it is enticing to project that should similar changes occur in women as occurred in men, the body mass of women forwards and backs will increase by approximately $3 \%$ and $7 \%$ over the next three RWC cycles.

Of course, many of these gains may already have been realised as a result of application of sports science and conditioning principles learnt from men's rugby and other sports, and the inclusion of Sevens in the Olympics will have accelerated talent search and high-performance systems to produce elite women players. ${ }^{21}$ This may be especially true for nations whose Olympic programmes and professional rugby structures are mature.

Generally, however, women's rugby is significantly smaller in size, both financially and in terms of player number, which creates possibilities for large effects should changes be made on either the talent identification or player development pathways that produce international women's players. That is, if investment into women's rugby improves the identification of more athletic women, or if professional environments like those that have driven men's increases in size are applied in more countries, then we would expect that the body mass and height of players in the sport will increase significantly in future.

\section{T1 versus T2 comparisons}

Our second important finding is that differences in body mass between $\mathrm{T} 1$ and T2 nations, while small in absolute terms, have persisted across the period from 1991 to 2019 , with some indications that the differences may be increasing. This is seen in the absolute differences in body mass between $\mathrm{T} 1$ and $\mathrm{T} 2$ players (table 1 , figure 1 ), in the proportion of both forwards and backs heavier and lighter than a historical reference value (figure 3), and in the evaluation of which teams are heaviest at RWCs (figure 4).

While the absolute differences in median and average body mass are small (table 1), we found that only $50 \%$ of T2 forwards were heavier than a reference value of $108 \mathrm{~kg}$ in 2019 , compared with $72 \%$ of $\mathrm{T} 1$ forwards (figure 3A). Of interest is that in 2007, the proportion of $\mathrm{T} 1$ and $\mathrm{T} 2$ forwards heavier than $108 \mathrm{~kg}$ 
was similar ( $58 \%$ for T1, $55 \%$ for T2), but the gap has increased at every tournament since, with the result that in 2019 , it reached its largest levels yet $(72 \%$ vs $50 \%$ ). This suggests that $\mathrm{T} 2$ nations are not producing heavier forwards and backs as readily as T1 nations, contributing to the $5 \mathrm{~kg}$ difference in median mass of forwards in 2019 (table 1).

A similar phenomenon exists when assessing the proportion of backs heavier than a reference value of $87 \mathrm{~kg}$ (figure 3B). This again suggests a relative inability of T2 teams to develop heavier backs, even though T2 backs median mass is similar to that of T1 nations (table 1). We also find that the proportion of T2 forwards and backs who are lighter than reference masses of $98 \mathrm{~kg}$ and $79 \mathrm{~kg}$, respectively, is greater than in $\mathrm{T} 1$ teams.

In effect, whether assessed relative to the median or these 1991 quartile reference points, T2 nations produce more lighter players, while T1 nations produce more heavier players (figure 3), and this disparity is emerging and increasing in 2019. PI teams feature among the heavier teams, though they are also classified as T2 nations. We chose to analyse these nations separately because it has long been perceived that their players are larger than those of other nations, which we confirm here.

We hypothesise that these differences are related to the previously described professional systems that develop rugby players. In particular, professional environments, often linked to well-developed school competitions in $\mathrm{T} 1$ nations, introduced many more young players to advanced training methods sooner. This increases their training age by the time they exit junior rugby, when they receive further advantages in high developed professional environments. This is expected to drive the physical development of players in ways that T2 nations have been unable to match.

Given the purported importance of size and strength to rugby performance and success, ${ }^{411}$ these disparities in body mass have implications for competitive parity. They may also contribute to welfare considerations for the smaller T2 players. It would be desirable to minimise the differences, and certainly to address what may be causing any body mass differences to become larger.

The same $\mathrm{T} 1$ versus $\mathrm{T} 2$ disparities are observed in women's rugby, where the heaviest $10 \%$ of teams are exclusively $\mathrm{T} 1$ nations, and the lightest teams are always T2 nations. Of significance is that in the most recent women's RWC in 2017, T1 forwards and backs were significantly heavier than T2 forwards and backs (figure 1). This is in part the result of an increase in body mass of T1 forwards since 2014, while T2 forwards and backs have stabilised or even decreased in body mass (figure 2). The proportion of T2 forwards and backs who are lighter than $74 \mathrm{~kg}$ and $64 \mathrm{~kg}$ has increased, while heavy forwards have decreased in T2 teams (figure 3C,D).
This may be of particular concern for parity between countries in the developing women's game. Given the purported importance of size to rugby performance outcomes, ${ }^{11}$ these enlarging disparities, while early, may entrench differences between established and developing nations and increase the barriers for success for developing T2 teams. This is particularly challenging for women's rugby, because if professional elements drive increases in player mass, among other attributes that enhance performance, as we have described above, then there is a risk that the vastly unequal timing and magnitude of introducing these elements may create performance differences that skew results dramatically in favour of early professional teams, and which will be very difficult to overcome in future.

Finally, our third important finding is to develop a set of reference ranges for men's and women's elite players, that may guide player identification, including at the talent identification stage, and the selection and management of players in elite performance systems.

\section{Limitations}

This study has some limitations. We are reliant on the body mass of players as reported by teams when their World Cup squads are announced and registered. There is a risk of both under-reporting and over-reporting, and we cannot independently verify the accuracy. However, given the large size of the cohorts, a strength of this study, and its longitudinal nature, we believe that any random inaccuracies in this regard will be minimised. As mentioned, we do not have the ability to explore whether the body mass reported is changing in terms of body composition, but acknowledge that stable body mass since 2011 does not necessarily imply that physical conditioning or composition of players is unchanging. Indeed, another possible explanation for the change in body mass of players early during professionalisation is doping of players, ${ }^{4}$ the effects of which would include increases in muscle mass and reductions in fat mass, and which may be present now. We do not have the necessary data to evaluate this possibility in the context of the aims of the present study.

We have not included height in this analysis, for the sake of clarity and to allow greater depth of analysis of body mass data, but the evolution of player height in conjunction with body mass would be worthy of future research, since it is arguably a significant contributor to the changes we describe here.

Our discussion of the findings is reliant on insights from elite coaches, who offer novel, and as yet untested theories to explain the results. These must be explored in future, with research to specifically refute or confirm the validity of the hypotheses we offer here. Finally, it must be acknowledged that analysis of players at a World Cup, held every 4 years, is itself a reflection of the wider game played at the professional and occasionally amateur levels around the world. While we analyse every tournament since 1991 and 2010 for men and women, respectively, we acknowledge that these tournaments are snapshots of 
a select group of players in comparison to the larger elite rugby-playing community, whose body masses may have varied differently over the analysis period.

\section{Conclusion}

In conclusion, we show here that men's player mass increased significantly soon after professional elements were introduced, but that the magnitude of these increases are now smaller in size and suggestive of the approach of an upper limit, perhaps driven by the evolution in match demands and the nature of play required in modern rugby. We show that women's rugby, early in its life cycle, has undergone some changes similar to those seen in men, though whether this continues into the future is uncertain. We also show that T2 nations are disadvantaged in size in both the men's and women's game, with lower median and mean mass of forwards and backs, and an apparently inability to produce as many players whose body mass lies above what would historically be considered heavy. This may have implications for the competitiveness of T2 nations, and requires assessment if gaps are not to become excessively large and material to performance.

Correction notice This article has been corrected since it first published. The provenance and peer review statement has been included.

\section{Twitter Ross Tucker@scienceofsport}

Contributors RT conceived the study and designed the study. RT, JB and CdC compiled the data and performed the analyses. RT drafted the article and all other authors revised it critically for important intellectual content. RT is the guarantor. All authors had full access to all of the data in the study and can take responsibility for the integrity of the data and the accuracy of the data analysis.

Funding The authors have not declared a specific grant for this research from any funding agency in the public, commercial or not-for-profit sectors.

Competing interests One of the authors (RT) is employed by World Rugby in roles of research and medicine. SL was the head coach of England at the 2015 Rugby World Cup. PD was the head coach of Namibia at the 2015 and 2019 Rugby World Cup. GS was the coach of the England Women's team at the 2014 Rugby World Cup.

\section{Patient consent for publication Not required.}

Ethics approval The research plan for this study was approved by the World Rugby Institutional Ethics committee (REF G-2003-00913). Since no player's medical information was used, and personal data were used only in a deanonymised form, and was available publicly through online sources, informed consent was not required from players in the relevant tournaments.

Provenance and peer review Not commissioned; externally peer reviewed.

Data availability statement Data are available upon reasonable request. Participant mass is publicly available in various forms including match day websites and other online sources. The majority of the data used for these analyses is available upon request to third parties.

Open access This is an open access article distributed in accordance with the Creative Commons Attribution Non Commercial (CC BY-NC 4.0) license, which permits others to distribute, remix, adapt, build upon this work non-commercially, and license their derivative works on different terms, provided the original work is properly cited, appropriate credit is given, any changes made indicated, and the use is non-commercial. See: http://creativecommons.org/licenses/by-nc/4.0/.

\section{ORCID iD}

Ross Tucker http://orcid.org/0000-0002-4294-4822

\section{REFERENCES}

1 Fuller CW, Brooks JHM, Cancea RJ, et al. Contact events in rugby Union and their propensity to cause injury. $\mathrm{Br} J$ Sports Med 2007;41:862-7.

2 World Rugby Game Analysis Department. RWC 2019 statistical report. world rugby. Available: https://playerwelfare.worldrugby.org/ content/getfile.php? $\mathrm{h}=4 \mathrm{~d} 3 \mathrm{c} 4500 \mathrm{fe} 6 \mathrm{a} 9 \mathrm{~d} 03 \mathrm{~b} 8 \mathrm{a} 482 \mathrm{cf589b54a6 \& p=}$ pdfs/gameanalysis/80.pdf\&d=RWC_Analysis_2019.pdf [Accessed 4 May 2020].

3 World Rugby Game Analysis Department. Six nations 2019 statistical report. world rugby. Available: https://playerwelfare.worldrugby. org/content/getfile.php?h=a2323cf95e58fefc1544e7a9db39c792\& $\mathrm{p}=$ pdfs/gameanalysis/79.pdf\&d=6_Nations_analysis_2019.pdf [Accessed 4 May 2020].

4 Hill NE, Rilstone S, Stacey MJ, et al. Changes in northern hemisphere male international rugby union players' body mass and height between 1955 and 2015. BMJ Open Sport Exerc Med 2018;4:e000459.

5 Fuller CW, Taylor AE, Brooks JHM, et al. Changes in the stature, body mass and age of English professional rugby players: a 10-year review. J Sports Sci 2013;31:795-802.

6 Fuller CW, Molloy MG, Bagate C, et al. Consensus statement on injury definitions and data collection procedures for studies of injuries in rugby Union. Br J Sports Med 2007;41:328-31.

7 Quarrie KL, Hopkins WG. Tackle injuries in professional rugby Union. Am J Sports Med 2008;36:1705-16.

8 Tierney GJ, Joodaki H, Krosshaug T, et al. Assessment of modelbased image-matching for future reconstruction of unhelmeted sport head impact kinematics. Sports Biomech 2018;17:33-47.

9 Kinnear C. Rugby: Welsh boss says limiting substitutions could ease injury crisis. NZ herald, 2017. Available: https://www.nzherald.co. $\mathrm{nz} /$ sport/news/article.cfm?c_id=4\&objectid=11964493 [Accessed 4 May 2020].

10 O'Connor B. Rugby needs to make itself smaller, weaker and less dangerous. The Irish times, 2019. Available: https://www.irishtimes. $\mathrm{com} / \mathrm{sport} /$ rugby/rugby-needs-to-make-itself-smaller-weaker-andless-dangerous-1.3803699 [Accessed 4 May 2020].

11 Sedeaud A, Marc A, Schipman J, et al. How they won rugby world cup through height, mass and collective experience. Br J Sports Med 2012;46:580-4.

12 Fuller CW, Taylor A, Kemp SPT, et al. Rugby world cup 2015: world rugby injury surveillance study. Br J Sports Med 2017;51:51-7.

13 Durandt J, Green M, Masimla H, et al. Changes in body mass, stature and BMI in South African elite U18 rugby players from different racial groups from 2002-2012. J Sports Sci 2018;36:477-84.

$14 \mathrm{Kemp}$ S, Brooks JHM, West S, et al. England professional rugby injury surveillance project. englandrugby.com. Available: http://www. englandrugby.com/mm/Document/General/General/01/32/25/17/ 1516_PRISP_Annual_Report_FINAL\%28withcontentspage\%29_ English.pdf [Äccessed 20 Jan 2017].

15 Tucker R, Raftery M, Kemp S, et al. Risk factors for head injury events in professional rugby Union: a video analysis of 464 head injury events to inform proposed injury prevention strategies. $\mathrm{Br} J$ Sports Med 2017;51:1152-7.

16 Tucker R, Raftery M, Fuller GW, et al. A video analysis of head injuries satisfying the criteria for a head injury assessment in professional rugby Union: a prospective cohort study. Br J Sports Med 2017;51:1147-51.

17 Cross MJ, Tucker R, Raftery M, et al. Tackling concussion in professional rugby Union: a case-control study of tackle-based risk factors and recommendations for primary prevention. $\mathrm{Br} J$ Sports Med 2019;53:1021-5.

18 Jones B, Weaving D, Tee J, et al. Bigger, stronger, faster, fitter: the differences in physical qualities of school and Academy rugby union players. J Sports Sci 2018;36:2399-404.

19 Tierney GJ, Richter C, Denvir K, et al. Could lowering the tackle height in rugby Union reduce ball carrier inertial head kinematics? J Biomech 2018;72:29-36.

20 Tierney GJ, Denvir K, Farrell G, et al. Does ball carrier technique influence tackler head injury assessment risk in elite rugby union? J Sports Sci 2019;37:262-7.

21 Tucker R. Rugby Sevens: Olympic debutante and research catalyst. Br J Sports Med 2016;50:638-9. 